\title{
Existence de dykes doléritiques anciens à composition de tholéiites continentales au sein de la province alcaline de la ligne du Cameroun. Implication sur le contexte géodynamique
}

\author{
Jean-Paul Vicat ${ }^{\mathrm{a}, \mathrm{b}}$, Ismaïla Ngounouno ${ }^{\mathrm{c}}$, André Pouclet ${ }^{\mathrm{b}, *}$ \\ a Département de géologie, université de N'Djamena, BP 1027, N’Djamena, Tchad \\ b Institut des sciences de la Terre, UMR 6113, université d'Orléans, BP 6759, 45067 Orléans cedex 2, France \\ c Département des sciences de la Terre, université de Ngaoundéré, BP 454 Ngaoundéré, Cameroun
}

Reçu le 6 novembre 2000 ; accepté le 23 janvier 2001

Présenté par Jean Aubouin

\begin{abstract}
Existence of old doleritic dykes of continental tholeiite composition, in the Cameroon Line alkaline province. Implication to the geodynamical context. In the Adamawa Plateau (Northern Cameroon), doleritic dykes belong to a magmatic activity which predated the Cenozoic alkaline volcanism of the Cameroon Line. They have the chemical composition of continental tholeiites with $\mathrm{Nb}$-Ta- and Ti-negative anomalies. This magmatism is interpreted as being derived from a sub-continental lithospheric source, which may have been contaminated during a former subduction event, linked to the Pan-African convergence. It is related to an early stage of continental break-up, which was contemporaneous or predated the Cretaceous tholeiitic magmatism of predominantly asthenospheric origin, which indicates a lithospheric thinning. ( 2001 Académie des sciences / Éditions scientifiques et médicales Elsevier SAS
\end{abstract}

Cameroon line / dolerites / low-Ti continental tholeiites / continental extension / lithospheric magma

Résumé - Sur le plateau de l'Adamaoua (Nord du Cameroun), des filons de dolérite traduisent une activité magmatique antérieure au volcanisme alcalin cénozoïque de la ligne du Cameroun. Leur composition chimique est celle de tholéiites continentales à anomalies négatives en $\mathrm{Nb}-\mathrm{Ta}$ et Ti. Ce magmatisme provient d'une source lithosphérique continentale qui aurait été contaminée lors de subductions liées à la convergence panafricaine. Il appartient à une phase précoce de fracturation continentale, contemporaine ou antérieure à celle du magmatisme tholéiitique crétacé, dont l'origine asthénosphérique indique le début de l'amincissement lithosphérique. @ 2001 Académie des sciences / Éditions scientifiques et médicales Elsevier SAS

ligne du Cameroun / dolérites / tholéiites continentales pauvres en Ti / extension continentale / magma lithosphérique

\section{Abridged version}

\section{Geological setting}

In Cameroon, the post Pan-African volcanism is mainly represented by the alkaline province of the Cameroon Line (figure 1) [5]. Some rare continental tholeiites are associated with the Early Palaeozoic tectonics [1] and with the development of Early Cretaceous basins [13]. In the Adamawa Plateau, South-East of Ngaoundéré, close to the Biden village (figures 2 and 3), we discovered do-

* Correspondance et tirés à part.

Adresse e-mail : gestion.isto@univ-orleans.fr (A. Pouclet). 
lerite dykes which cross-cut the Palaeozoic granites. The Adamawa Plateau is made up of Pan-African granites and gneisses intruded by some granitic and syenitic bodies of probably Ordovician age [15]. It suffered intense fracturation, during Mesozoic and Early Cenozoic time, along $\mathrm{N} 70^{\circ}$ and $\mathrm{N} 130^{\circ}-140^{\circ}$ fault systems leading to the formation of the Cretaceous troughs and to the Mio-Pliocene volcanic activity [7]. The dolerite dykes of Biden are subvertical and 0.5 to $1.5 \mathrm{~m}$ thick. They are trending $\mathrm{N} 10^{\circ}-$ $30^{\circ}, \mathrm{N} 70^{\circ}$ and $\mathrm{N} 135^{\circ}$, in the same directions as for the Pan-African and the post-Pan-African tectonics [14]. They are clearly post-Ordovician and may be related to a Late Palaeozoic poorly defined tectonic event or to the wellknown Cretaceous extensive stage.

\section{Petrography and geochemistry}

Dolerites are coarse-grained to fine-grained from the centre to the margin of dykes. The texture is intergranular to sub-ophitic. Mineralogic association consists of zoned phenocrysts of diopside to calcic augite $(\mathrm{Ca} \%$ : 44-39, $\mathrm{Mg} \%$ : 46-36, $\mathrm{Fe}_{\mathrm{t}}+\mathrm{Mn} \%$ : 14-28), syncrystallized plagioclase (An52-18, Ab43-75, Or5-7) and actinolite $\left(X_{\mathrm{Mg}}=0.65-0.84\right)$ corresponding to ouralitized pyroxene. The groundmass contains microcrysts of sodic plagioclase (An15 Ab76 Or9), clinopyroxene (Ca\%: 40, $\mathrm{Mg} \%$ : 37, $\left.\mathrm{Fe}_{\mathrm{t}} / \mathrm{Mn} \%: 23\right)$, biotite $\left(X_{\mathrm{Fe}}=0.37\right)$, Ti-magnetite, ilmenite, and interstitial quartz. Some rocks contain phenocrysts of enstatite (Ca\%: 3.5, $\mathrm{Mg} \%: 69, \mathrm{Fe}_{\mathrm{t}}+\mathrm{Mn} \%$ : $\left.27.5, X_{\mathrm{Mg}}=0.72\right)$ often mantled by clinopyroxene $(\mathrm{Ca} \%$ : 39, $\mathrm{Mg} \%: 46, \mathrm{Fe}_{\mathrm{t}}+\mathrm{Mn} \%$ : 15). This orthopyroxene is chemically different from that analysed in the mantle xenoliths [11] and is of magmatic origin. The paragenesis and the order of crystallization are indicative of a low waterpressure condition and of a tholeiitic to transitional feature.

The ten analysed samples (table) have moderately evolved compositions $\left(\mathrm{SiO}_{2}=58.6-59.5, \mathrm{DI}=64-66, \mathrm{Mg} \#=\right.$ 44-47); they are oversaturated (norm Qtz\% $=9-10$ ), and alkaline potassic (figure 4). The primitive mantle normalized patterns (figure 5) show important incompatible element fractionation $\left(43.0<\mathrm{La}_{\mathrm{N}} / \mathrm{Yb}_{\mathrm{N}}<52.4\right)$ and strong
$\mathrm{Nb}-$, Ta- and Ti-negative anomalies $\left(\mathrm{Nb}_{\mathrm{N}} / \mathrm{La}_{\mathrm{N}}=0.28\right.$, $\left.\mathrm{Ti}_{\mathrm{N}} / \mathrm{Tb}_{\mathrm{N}}=0.70\right)$. These patterns are those of active margin lavas and of low-Ti continental flood basalts (low-Ti CFB). Enrichment in alkalies and incompatible elements and the biotite occurrence are consistent with a composition of an evolved tholeiitic magma.

\section{Discussion and conclusion}

The chemical composition of the Biden dolerites is clearly different from that of other mafic lavas of the Cameroon Line area. The geographically nearest MioPliocene basalts from Ngaoundéré (figure 2) are exclusively alkaline [16] (figures 5 and 6 ). The Cretaceous dolerites from Ngaoundal, located $80 \mathrm{~km}$ south of Biden are tholeiitic, with $\mathrm{Th} / \mathrm{Ta}, \mathrm{Th} / \mathrm{Nb}$ and $\mathrm{Nb} / \mathrm{La}$ ratios of common continental tholeiites (figures 4-6) and particularly of initial rift tholeiites [10], the source of which involves an asthenospheric component. Similar chemical features characterize the tholeiitic basalts from the Southern Benue Trough (Cenomanian to Santonian) and from the Northern Benue Trough (Late Jurassic to Albian) [4], as well as the tholeiites of the Garoua area associated with the Palaeozoic basins of Balché and Mangbaï [1].

The chemical composition of the Biden dolerites is therefore original, owing to their $\mathrm{Nb}-$, Ta-, and Ti-negative anomalies. The same chemical features are known in the low-Ti CFB of the Karoo and the Ferrar magmatic Mesozoic Province [9] (figures 5-6). The anomalies are interpreted as a result of contamination of the sub-continental mantle by subducted sediments [9]. The Biden dolerites being more evolved, they are richer in incompatible element, but their patterns are close to that of Ferrar tholeiites.

It is concluded that the Biden dolerites are low-Ti tholeiites generated from a sub-continental lithospheric mantle similar to that of the Karoo and Ferrar Province. Contamination of this mantle may have occurred during the Pan-African subduction events [20]. These dolerites, devoid of an asthenospheric component in their source, can be attributed either to the Cretaceous extensive event but located far to the rift axis, or to an early stage of the continental breaking of the Cameroon Line area.

\section{Introduction}

$\mathrm{Au}$ Cameroun le volcanisme post-panafricain est essentiellement représenté par la ligne du Cameroun (figure 1), de nature alcaline [5]. Quelques rares tholéiites continentales sont signalées dans les fossés ordoviciens à dévoniens du Nord-Cameroun [1] et dans les fossés crétacés [15]. Sur le plateau de l'Adamaoua, nous avons découvert des dykes de dolérites post-panafricains, au sud-est de Ngaoundéré, aux environs du village de Biden (figures 2 et 3 ). Ces roches à composition de tholéiites continentales se singularisent par de fortes anomalies négatives en $\mathrm{Nb}-$ Ta et Ti.

\section{Contexte géologique et gisement}

Le plateau de l'Adamaoua, d'altitude moyenne $1100 \mathrm{~m}$, est un horst incliné vers le sud, limité au nord par la faille de Ngaoundéré et au sud par la faille de Mbéré-Djérem (figure 2). Il est essentiellement constitué de granito-gneiss panafricains, recoupés par des plutons de granites et de syénites postpanafricains, d'âge probablement Ordovicien [17]. Ce 


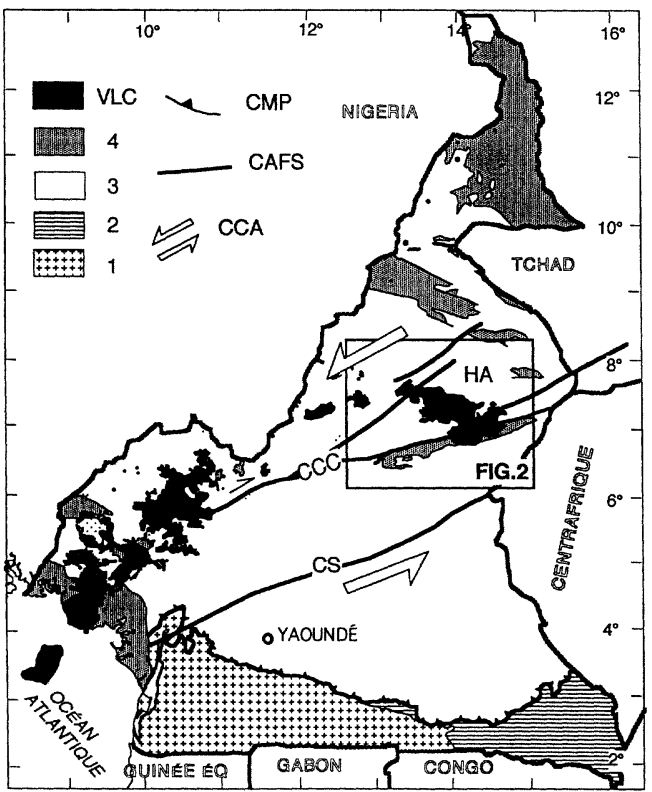

Figure 1. Croquis géologique du Cameroun. 1 : groupe archéen du Ntem $; \mathbf{2}$ : couverture protérozoïque $; \mathbf{3}$ : chaîne panafricaine $; \mathbf{4}$ : dépôts phanérozoïques. VLC : volcanisme et plutonisme de la ligne du Cameroun; CMP : chevauchement majeur panafricain ; CAFS : système de faille d'Afrique centrale (CS, cisaillement de la Sanaga ; CCC, cisaillements centro-camerounais); CCA : mouvement du méga-cisaillement cénozoïque à actuel ; HA : horst de l'Adamaoua.

Figure 1. Geological sketch map of Cameroon. 1: Archaean Ntem Group; 2: Proterozoic cover; 3: Pan-African belt; 4: Phanerozoic deposits. VLC: Cameroon Volcanic Line; CMP: Pan-African major thrust; CAFS: Central African fault system (CS, Sanaga strike-slip fault; CCC, Central Cameroon strike-slip fault); CCA: Cenozoic to Present mega-shear-zone motion; HA: horst de l'Adamaoua.

horst est intensément fracturé selon les directions $\mathrm{N} 70^{\circ} \mathrm{E}$ et $\mathrm{N} 130^{\circ}-140^{\circ} \mathrm{E}$. Au Paléozoïque inférieur, les petits bassins de Balché et de Mangbaï [1] s'ouvrent au nord de l'Adamaoua. Au Mésozoïque et au Cénozoïque, une intense réactivation des failles du substratum panafricain est à l'origine de la formation du système de rifts d'Afrique centrale et occidentale [8], auquel appartiennent les bassins crétacés de Vina et de Mbéré-Djérem (figure 2). Des formations volcaniques, d'âge essentiellement Mio-Pliocène [7], recouvrent partiellement l'ensemble des formations précédentes.

Un essaim de dykes de dolérites recoupe les granitoïdes du Paléozoïque inférieur à moyen aux environs du village de Biden, à $3 \mathrm{~km}$ au sud-est de Ngaoundéré (figure 3). Les filons, d'épaisseur comprise entre 0,5 à $1,5 \mathrm{~m}$, occupent les plans de fracturation des granites encaissants. Ils présentent des pendages forts $\left(>70^{\circ}\right)$ à verticaux. Leurs orientations sont $\mathrm{N} 10^{\circ}-30^{\circ}, \mathrm{N} 70^{\circ}$ et $135^{\circ}$, directions correspondant aux structures panafricaines du Nord du Cameroun [14]. On note un léger métamorphisme de contact des granites encaissants. Le contact très net indique une mise en place

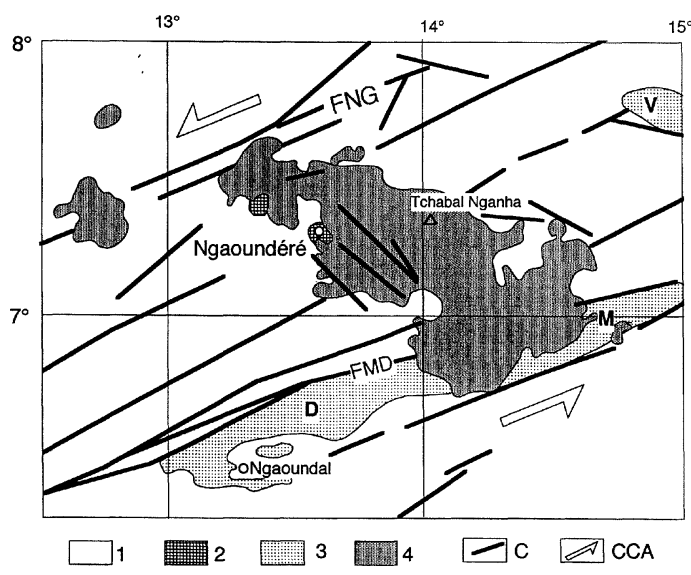

Figure 2. Croquis géologique de la région de Ngaoundéré. 1 : domaine orogénique panafricain ; $\mathbf{2}$ : granites post-tectoniques, d'âge probablement Ordovicien; $\mathbf{3}$ : fossés crétacés ( $\mathbf{V}$, Vina; $\mathbf{M}$, Mbéré ; $\mathbf{D}$, Djérem) ; $\mathbf{4}$ : volcanisme mio-pliocène. $\mathbf{C}$ : grands décrochements (FMD : faille de Mbéré-Djérem; FNG : faille de Ngaoundéré). CCA : mouvement du méga-cisaillement cénozoïque à actuel.

Figure 2. Geological sketch map of the Ngaoundéré zone. 1: PanAfrican orogenic domain; 2: post-tectonic granites probably of Ordovician age; 3: Cretaceous rifts; 4: Mio-Pliocene volcanism; C: Central Cameroon strike-slip fault (FMD, Mbéré-Djérem fault; FNG, Ngaoundéré fault); CCA: Cenozoïc to Present mega-shearzone motion.

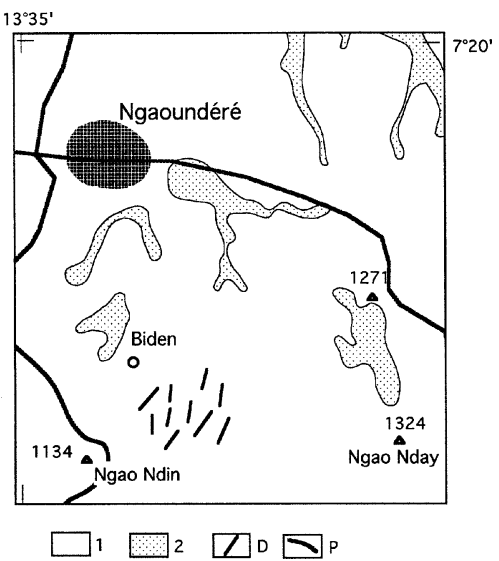

Figure 3. Localisation des dolérites de Biden. 1 : granite postpanafricain ; 2 : Quaternaire. D : dolérites ; $\mathbf{P}$ : principales pistes.

Figure 3. Biden dolerites location map. 1: Post-Pan-African granite; 2: Quarternary deposits. D: dolerites; P: main roads.

dans un encaissant froid. Ces dykes sont nettement post-ordoviciens. Ils peuvent être liés à une extension du Paléozoïque supérieur assez mal définie au NordCameroun ou à l'extension majeure du Crétacé.

\section{Pétrographie}

Les dolérites présentent un grain grossier au cœur des dykes et un grain fin en bordure, avec un faciès 
de trempe développé sur quelques centimètres. La texture est intergranulaire à subophitique.

Les dolérites communes à gros grain contiennent des phénocristaux zonés de diopside à augite calcique $\left(\mathrm{Ca} \%\right.$ : 44-39, $\mathrm{Mg} \%: 46-36, \mathrm{Fe}_{\mathrm{t}}+\mathrm{Mn} \%$ : 14-28), de plagioclase (An52-18, Ab43-75, Or5-7) et d'actinote $\left(X_{\mathrm{Mg}}=0,65-0,84\right)$ qui apparaît comme le produit d'ouralitisation des cristaux d'augite. Le plagioclase est souvent en intercroissance avec le clinopyroxène (cristallisation cotectique). La mésostase renferme des microcristaux de plagioclase sodique (An15, Ab76, Or9), de clinopyroxène $(\mathrm{Ca} \%: 40$, $\mathrm{Mg} \%: 37$, Fet $+\mathrm{Mn} \%: 23)$, de biotite ferrifère $\left(X_{\mathrm{Fe}}=0,37\right)$ de titanomagnétite, d'ilménite en amas et de quartz interstitiel.

Plusieurs dolérites, notamment celles à grain fin, montrent des phénocristaux d'enstatite $(\mathrm{Ca} \%: 3,5$, $\left.\mathrm{Mg} \%: 69, \mathrm{Fe}_{\mathrm{t}}+\mathrm{Mn} \%: 27,5 ; X_{\mathrm{Mg}}=0,72\right)$, souvent auréolés d'augite calcique $(\mathrm{Ca} \%: 39, \mathrm{Mg} \%$ : $\left.46, \mathrm{Fe}_{\mathrm{t}}+\mathrm{Mn} \%: 15\right)$. Les teneurs moyennes en $\mathrm{CaO}$ $(1,7 \%)$ et $\mathrm{FeO}_{\mathrm{t}}(17,2 \%)$ de l'enstatite sont plus élevées que celles observées dans les orthopyroxènes mantelliques et, plus particulièrement, dans ceux des enclaves de lherzolites à spinelle et plagioclase, exhumées par les basanites cénozoïques du plateau de l'Adamaoua [11]. Ce sont bien des orthopyroxènes magmatiques, témoins d'une phase précoce de cristallisation.

L'ordre d'apparition des principaux minéraux (Opxplagioclase, augite et oxydes de fer-titane-biotite et titanite-quartz) traduit une cristallisation sous de faibles pressions d'eau et caractérise une affinité tholéiitique à transitionnelle.

\section{Géochimie}

Nous avons analysé dix échantillons provenant de différents filons (tableau). La composition chimique est celle de roches intermédiaires $\left(\mathrm{SiO}_{2}=58,6\right.$ $59,5 \%$ ), différenciées ( $\mathrm{DI}=64-66, \mathrm{Mg} \#=44-47$ ) et à quartz normatif (9-10\%). Dans le diagramme TAS (figure 4), les dolérites sont des trachyandésites ou des latites $\left(\mathrm{K}_{2} \mathrm{O}>\mathrm{Na}_{2} \mathrm{O}-2\right)$, à la limite des domaines alcalins et sub-alcalins [13]. Les profils de terres rares sont fractionnés $\left(43,0<\mathrm{La}_{\mathrm{N}} / \mathrm{Yb}_{\mathrm{N}}<52,4\right)$. Les profils normalisés au manteau primitif [18] montrent un enrichissement en éléments les plus incompatibles, avec des anomalies négatives en $\mathrm{Nb}-\mathrm{Ta}\left(\mathrm{Nb}_{\mathrm{N}} / \mathrm{La}_{\mathrm{N}}=0,28\right)$ et en $\mathrm{Ti}\left(\mathrm{Ti}_{\mathrm{N}} / \mathrm{Tb}_{\mathrm{N}}=0,70\right)$ (figure 5). Ces anomalies se retrouvent dans les laves des marges actives et dans les basaltes de plateaux pauvres en titane (low-Ti CFB). Globalement, ces compositions, avec des teneurs assez élevées en alcalins et en éléments incompatibles, ainsi que l'apparition de biotite, correspondent à des produits différenciés d'un magma tholéiitique.

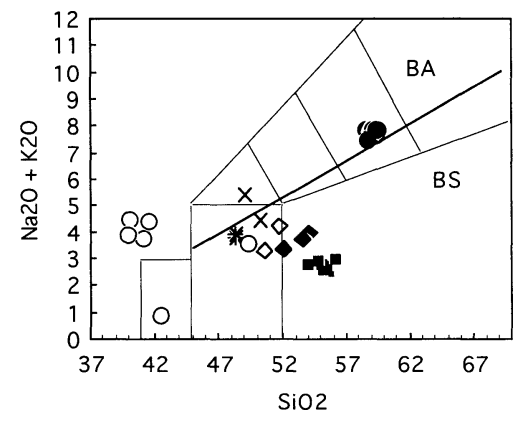

Figure 4. Diagramme TAS [12]. Limite des basaltes alcalins (BA) et subalcalins (BS) d'après [13]. Cercles noirs : dolérites de Biden (nouvelles analyses, IN27 à IN36). Cercles vides : basalte alcalin miopliocène de Ngaoundéré (analyses inédites). Étoiles : basaltes tholéiitiques crétacés de Ngaoundal (analyses inédites). Losanges vides : basaltes tholéiitiques (fin Jurassique à Albien) de la Haute Bénoué [4]. Losanges pleins : basaltes tholéiitiques (Cénomanien à Santonien) de la Basse Bénoué [4]. $\times$ : tholéiites des bassins paléozoïques de Balché et Mangbaï (Silurien supérieur à Dévonien supérieur) [1]. Carrés noirs : low Ti-CFB d'Australie (170-190 Ma) [9].

Figure 4. TAS diagram [12]. Boundary of alkali basalts and subalkaline basalts after [13]. Solid circles: dolerites from Biden (present work, IN27 to IN36). Open circles: Mio-Pliocene alkali basalts from Ngaoundéré (unpublished data). Stars: Cretaceous tholeiitic basalts from Ngaoundal (unpublished data). Open diamonds: Jurassic to Albian tholeiitic basalts from Southern Benue Trough [4]. Solid diamonds: Cenomanian to Santonian tholeiitic basalts of Northern Benue Trough [4]. $\times$ : tholeiites from Palaeozoic basins of Balché and Mangbaï (Late Silurian to Late Devonian) [1]. Solid squares: low Ti-Continental Flood Basalts from Australia (170-190 Ma) [9].

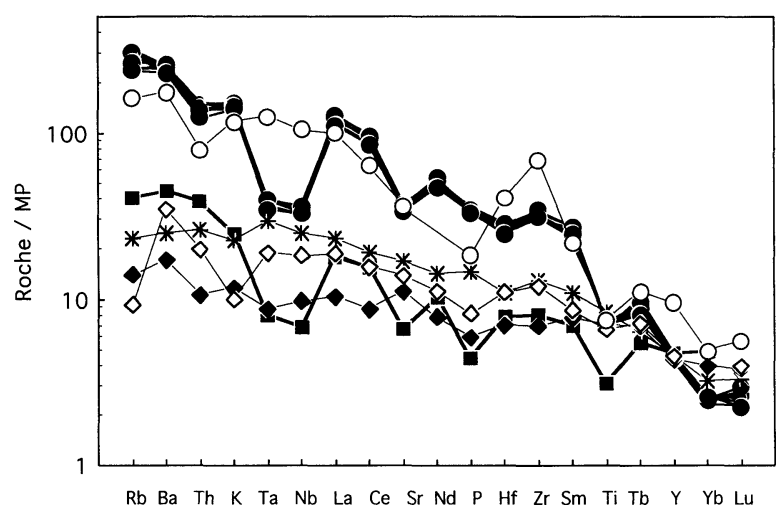

Figure 5. Profils normalisés au manteau primitif [18]. Mêmes symboles que pour la figure 4. Dolérites de Biden (échantillons IN27 à IN36, nouvelles analyses). Basalte alcalin mio-pliocène de Ngaoundéré (analyse inédite). Basalte tholéiitique crétacé de Ngaoundal (analyse inédite). Basalte tholéiitique de la Haute Bénoué (échantillon 153B1) et de la Basse Bénoué (échantillon 86B1) [4]. Low Ti-CFB de la province magmatique de Ferrar (moyenne de six analyses) [9].

Figure 5. Primitive mantle-normalized patterns [18]. Same symbols as for figure 4. Dolerites from Biden (samples IN27 to IN36, present work). Mio-Pliocene alkali basalt from Ngaoundéré (unpublished data). Cretaceous tholeiitic basalts from Ngaoundal (unpublished data). Tholeiitic basalt from Southern Benue Trough (sample 153B1) and of Northern Benue Trough (sample 86B1) [4]. Low Ti-CFB from the Ferrar magmatic province (average of six analyses) [9]. 
Tableau. Composition chimique des dolérites de Biden. Éléments majeurs en pourcentage pondéral, éléments mineurs et traces en ppm. Analyses réalisées au CRPG (Nancy) par ICP (éléments majeurs et mineurs) et ICP-MS (éléments en traces). DI : indice de différenciation de [19]; Mg\# : $\mathrm{Mg} / \mathrm{Mg}+\mathrm{Fe}^{\prime \prime}$.

Table. Chemical composition of the Biden dolerites. Major elements (weight \%), minor and trace elements (ppm). Analyses performed at the CRPG (Nancy) by ICP (major and some minor elements) and ICP-MS (trace elements). DI: differentiation index after [19]; Mg\#: Mg/Mg + Fe" .

\begin{tabular}{|c|c|c|c|c|c|c|c|c|c|c|}
\hline Ref. & IN27 & IN31 & IN32 & IN34 & IN35 & IN28 & IN29 & IN30 & IN33 & IN36 \\
\hline $\mathrm{SiO}_{2}$ & 59,22 & 58,56 & 59,36 & 58,93 & 59,11 & 59,48 & 58,88 & 58,86 & 59,39 & 58,68 \\
\hline $\mathrm{TiO}_{2}$ & 1,54 & 1,52 & 1,52 & 1,58 & 1,54 & 1,55 & 1,55 & 1,56 & 1,57 & 1,53 \\
\hline $\mathrm{Al}_{2} \mathrm{O}_{3}$ & 14,95 & 14,95 & 14,92 & 14,87 & 14,96 & 15,21 & 14,94 & 15,05 & 15,17 & 14,92 \\
\hline $\mathrm{Fe}_{2} \mathrm{O}_{3}$ & 6,95 & 6,80 & 6,99 & 7,19 & 6,94 & 7,10 & 7,09 & 7,16 & 7,11 & 7,13 \\
\hline $\mathrm{MnO}$ & 0,09 & 0,08 & 0,08 & 0,08 & 0,09 & 0,09 & 0,09 & 0,09 & 0,08 & 0,09 \\
\hline $\mathrm{MgO}$ & 2,61 & 2,62 & 2,61 & 2,77 & 2,60 & 2,67 & 2,83 & 2,75 & 2,52 & 2,74 \\
\hline $\mathrm{CaO}$ & 4,20 & 4,14 & 4,34 & 4,20 & 4,24 & 4,32 & 4,46 & 4,34 & 4,30 & 4,31 \\
\hline $\mathrm{Na}_{2} \mathrm{O}$ & 3,26 & 3,30 & 3,26 & 3,24 & 3,28 & 3,47 & 3,32 & 3,36 & 3,49 & 3,23 \\
\hline $\mathrm{K}_{2} \mathrm{O}$ & 4,64 & 4,53 & 4,40 & 4,55 & 4,60 & 4,40 & 4,23 & 4,18 & 4,37 & 4,23 \\
\hline $\mathrm{P}_{2} \mathrm{O}_{5}$ & 0,75 & 0,74 & 0,70 & 0,71 & 0,79 & 0,78 & 0,73 & 0,73 & 0,75 & 0,72 \\
\hline $\mathrm{PF}$ & 1,81 & 1,68 & 1,76 & 1,84 & 1,91 & 1,08 & 1,70 & 1,45 & 1,20 & 1,93 \\
\hline Total & 100,02 & 98,92 & 99,94 & 99,96 & 100,06 & 100,15 & 99,82 & 99,53 & 99,95 & 99,51 \\
\hline $\mathrm{Ba}$ & 1740 & 1802 & 1791 & 1734 & 1624 & 1765 & 1734 & 1715 & 1820 & 1607 \\
\hline $\mathrm{Ce}$ & 168,4 & 177,8 & 173,8 & 165,4 & 155,5 & 166,5 & 166,3 & 165,9 & 172,8 & 152,3 \\
\hline $\mathrm{Co}$ & 17,2 & 18,3 & 19,9 & 19,5 & 16,9 & 15,5 & 17,7 & 19,1 & 18,5 & 19,6 \\
\hline $\mathrm{Cr}$ & 50,4 & 58,1 & 56,3 & 56,1 & 54,9 & 58,9 & 60,4 & 54,0 & 50,8 & 62,2 \\
\hline Dy & 4,71 & 5,03 & 4,82 & 4,70 & 4,36 & 4,95 & 4,94 & 4,86 & 4,97 & 4,34 \\
\hline $\mathrm{Er}$ & 1,79 & 1,77 & 1,86 & 1,70 & 1,67 & 1,87 & 1,82 & 1,80 & 1,69 & 1,57 \\
\hline $\mathrm{Eu}$ & 2,94 & 3,04 & 2,85 & 2,84 & 2,70 & 3,14 & 2,94 & 3,04 & 2,99 & 2,63 \\
\hline $\mathrm{Ga}$ & 23,2 & 26,8 & 26,5 & 25,3 & 24,4 & 23,2 & 24,4 & 24,8 & 26,7 & 24,6 \\
\hline $\mathrm{Gd}$ & 8,22 & 9,09 & 8,57 & 8,75 & 7,18 & 8,81 & 8,39 & 9,05 & 8,52 & 7,12 \\
\hline $\mathrm{Hf}$ & 8,42 & 8,31 & 8,04 & 8,45 & 7,58 & 8,87 & 8,07 & 8,60 & 8,72 & 7,66 \\
\hline $\mathrm{La}$ & 82,93 & 87,93 & 88,81 & 85,54 & 78,49 & 85,63 & 85,33 & 85,03 & 88,41 & 76,69 \\
\hline $\mathrm{Lu}$ & 0,19 & 0,18 & 0,19 & 0,20 & 0,17 & 0,21 & 0,19 & 0,19 & 0,22 & 0,17 \\
\hline $\mathrm{Nb}$ & 23,52 & 25,7 & 25,47 & 25,35 & 22,98 & 24,16 & 23,78 & 23,85 & 25,48 & 23,26 \\
\hline $\mathrm{Nd}$ & 68,19 & 70,73 & 71,59 & 69,45 & 65,19 & 71,22 & 67,91 & 69,92 & 73,13 & 63,61 \\
\hline $\mathrm{Ni}$ & 26,5 & 27,6 & 29,2 & 29,2 & 27,9 & 26,9 & 31,6 & 29,5 & 28,1 & 34,4 \\
\hline $\mathrm{Pb}$ & 17,5 & 18,5 & 16,9 & 17,4 & 17,1 & 18,1 & 17,2 & 16,3 & 16,9 & 15,2 \\
\hline $\operatorname{Pr}$ & 18,73 & 19,18 & 19,37 & 18,69 & 17,04 & 17,71 & 18,09 & 18,96 & 19,31 & 17,00 \\
\hline $\mathrm{Rb}$ & 183,6 & 198,6 & 192,9 & 194,3 & 188,9 & 153,8 & 156,0 & 159,5 & 169,0 & 151,9 \\
\hline $\mathrm{Sm}$ & 10,80 & 12,21 & 12,22 & 11,08 & 10,62 & 11,38 & 11,97 & 11,39 & 12,11 & 10,90 \\
\hline $\mathrm{Sr}$ & 708 & 793 & 777 & 756 & 730 & 745 & 729 & 736 & 784 & 714 \\
\hline $\mathrm{Ta}$ & 1,62 & 1,61 & 1,63 & 1,64 & 1,49 & 1,64 & 1,63 & 1,66 & 1,64 & 1,42 \\
\hline $\mathrm{Tb}$ & 0,98 & 1,09 & 1,04 & 0,97 & 0,94 & 1,02 & 0,98 & 1,03 & 1,04 & 0,88 \\
\hline $\mathrm{Th}$ & 12,82 & 12,94 & 13,15 & 11,85 & 11,32 & 12,68 & 11,92 & 12,05 & 11,65 & 10,61 \\
\hline $\mathrm{V}$ & 105 & 116 & 119 & 120 & 113 & 107 & 106 & 107 & 116 & 110 \\
\hline $\mathrm{Y}$ & 18,9 & 21,5 & 20,8 & 20,3 & 19,2 & 19,6 & 19,0 & 19,7 & 21,0 & 20,2 \\
\hline $\mathrm{Yb}$ & 1,21 & 1,24 & 1,30 & 1,26 & 1,14 & 1,21 & 1,29 & 1,29 & 1,21 & 1,28 \\
\hline $\mathrm{Zr}$ & 342 & 378 & 369 & 364 & 343 & 355 & 336 & 357 & 380 & 346 \\
\hline DI & 65,74 & 65,68 & 65,01 & 64,83 & 65,65 & 65,12 & 63,82 & 63,99 & 65,49 & 63,89 \\
\hline $\mathrm{Mg} \#$ & 45,77 & 46,42 & 45,64 & 46,42 & 45,7 & 45,81 & 47,3 & 46,35 & 44,34 & 46,34 \\
\hline
\end{tabular}

\section{Interprétation géotectonique et discussion}

La composition des dolérites de Biden est différente de celle des autres magmas basiques connus dans la région. Les basaltes mio-pliocènes de Ngaoundéré, géographiquement les plus proches (figure 2), ont en effet une composition alcaline [16] qui ex- clut tout lien génétique avec les dolérites de Biden (figures 5 et 6 ). Les dolérites crétacées de Ngaoundal, localisées à $80 \mathrm{~km}$ au sud de Biden (figure 2) ont une composition tholéiitique, avec des rapports $\mathrm{Th} / \mathrm{Ta}$ compris entre 2 et 5 , correspondant à des tholéiites continentales [3], mais présentent un enrichissement relatif en $\mathrm{Nb}$ caractéristique de la plupart des tholéiites de rift initial [10], d'origine en partie asthé- 
J.-P. Vicat et al. / C. R. Acad. Sci. Paris, Sciences de la Terre et des planètes / Earth and Planetary Sciences 332 (2001) 243-249

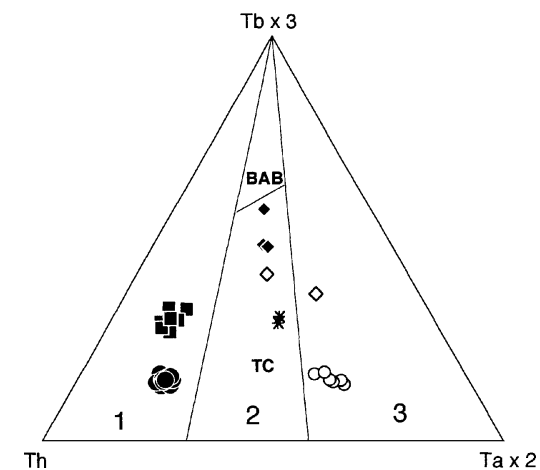

Figure 6. Diagramme Th-Tbx3-Tax 2 de [3]. Mêmes symboles que pour la figure 4. 1 : domaine orogénique d'arc; 2 : domaine intermédiaire des tholéiites continentales (TC) et des basaltes des bassins d'arrière-arc $(\mathrm{BAB}) ; \mathbf{3}$ : domaine anorogénique des tholéiites de dorsales et des basaltes alcalins intra-plaques. Noter la position des dolérites de Biden et des tholéiites de la province de Ferrar dans le domaine orogénique, malgré leurs localisations intraplaques, en raison de leurs anomalies négatives en Ta.

Figure 6. Th-Tbx3-Tax2 diagram [3]. Same symbols as for figure 4. 1: arc-related orogenic series; 2 : intermediate series of continental tholeiites (TC) and of back-arc basin basalts (BAB); 3: anorogenic series of oceanic ridges and intraplate alkaline basalts. Note the position of the Biden dolerites and of the Ferrar Province tholeiites in the orogenic domain, in spite of their intraplate setting, due to their Ta-negative anomaly.

nosphérique (figures 4-6). Plus à l'ouest, les basaltes tholéiitiques de la Basse Bénoué (Cénomanien à Santonien) et de la Haute-Bénoué (fin Jurassique à Albien) [4] ressemblent à ceux de Ngaoundal. Au NordCameroun, dans la région de Garoua, les tholéiites des bassins paléozoïques de Balché et Mangbaï [1] appartiennent aussi aux domaines continentaux d'après les teneurs en $\mathrm{Y}, \mathrm{Nb}$ et La, mais sans anomalies importantes [2].

La composition chimique des dolérites de Biden est donc originale par les anomalies en $\mathrm{Nb}$-Ta-Ti. Elles diffèrent des tholéiites continentales, dont les anomalies négatives en $\mathrm{Nb}-\mathrm{Ta}$, plus modérées, sont attribuées à la contamination crustale des magmas en route vers la surface [6]. Les basaltes de plateaux pauvres en $\mathrm{Ti}$ (low Ti-CFB), particulièrement ceux de la province du Karoo et de Ferrar en Australie [9] présentent au contraire des anomalies négatives importantes en $\mathrm{Nb}-\mathrm{Ta}$ (figure 5). Ces anomalies sont attribuées à une contamination de la source mantellique par des fluides issus d'une écorce subductée [9]. Les profils des dolérites de Biden sont tout à fait semblables à ceux des low $T i-C F B$ de la province Ferrar (figure 5), mais, étant plus différenciés, ils sont plus enrichis en éléments incompatibles. L'appauvrissement en Ta leur confère, comme les low Ti-CFB, un caractère orogénique dans les diagrammes discriminants, comme le diagramme Th- $\mathrm{Ta}-\mathrm{Tb}$ [3] (figure 6), ce qui contredit une mise en place dans un contexte intraplaque post-panafricain.

\section{Conclusion}

Les dolérites anciennes de Biden, dans l'Adamaoua, montrent une signature chimique originale dans la province alcaline de la ligne du Cameroun. $\mathrm{Ce}$ sont des tholéiites de type CFB (Continental Flood Basalt) pauvres en $\mathrm{TiO}_{2}$ et typiquement d'origine lithosphérique. La composition de la lithosphère dans ce secteur rappelle celle de la province tholéiitique continentale, pauvre en titane, du Karoo et de Ferrar. Dans le cas de l'Adamaoua, cette composition peut s'expliquer par la contamination des sources lithosphériques, lors des subductions liées à l'orogenèse panafricaine de la chaîne des Oubanguides [20]. Les autres tholéiites du Crétacé de la ligne du Cameroun sont des tholéiites continentales de type tholéiites de rift initial, qui montrent la participation d'une composante asthénosphérique. Les dolérites de Biden, uniquement lithosphériques, seraient, soit contemporaines, mais situées en dehors de l'axe du rift, soit plus précoces, et marqueraient alors le début de l'apparition des contraintes extensives. Par la suite, le développement de la tectonisation s'est accompagnée d'une contribution croissance de l'asthénosphère dans le magmatisme.

Remerciements. Nous remercions Christian Coulon pour ses corrections très efficaces, qui nous ont permis d'améliorer notre texte.

\section{Références}

[1] Béa A., Cochemé J.-J., Trompette R., Affaton P., Soba D., Sougy J., Grabens d'âge Paléozoïque inférieur et volcanisme tholéiitique associé dans la région de Garoua au Nord-Cameroun, J. Afr. Earth Sci. 10 (4) (1990) 657-667.

[2] Cabanis B., Lecolle M., Le diagramme La/10- $\mathrm{Y} / 15-\mathrm{Nb} / 8$ : un outil pour la discrimination des séries volcaniques et la mise en évidence des processus de mélange et/ou de contamination crustale, C. R. Acad. Sci. Paris, série II 309 (1989) 2023-2029.

[3] Cabanis B., Thiéblemont D., La discrimination des tholéiites continentales et des basaltes arrière-arc. Proposition d'un nouveau diagramme Th-Tbx3-Tax2, Bull. Soc. géol. France 86 (4) (1988) 927-935.

[4] Coulon C., Vidal P., Dupuy C., Baudin P., Popoff M., Maluski H., Hermitte D., The Mesozoic to Early Cenozoic magmatism of the Benue rough (Nigeria); geochemical evidence for the involvment of the St Helena plume, J. Petrol. 37 (6) (1996) 1341-1358.

[5] Déruelle B., Moreau C., Nkoumbou C., Kambou R., Lissom J., Njongfang E., Ghogomu R.T., Nono A., The Cameroon line: a review, in: Kampunzu A.B., Lubala R.T. (Eds.), Magmatism in extensional settings, The Phanerozoic African Plate, Springer Verlag, Berlin, 1991, pp. 274-327. 
J.-P. Vicat et al. / C. R. Acad. Sci. Paris, Sciences de la Terre et des planètes / Earth and Planetary Sciences 332 (2001) 243-249

[6] Dupuy C., Dostal J., Trace element geochemistry of some continental tholeiites, Earth Planet. Sci. Lett. 67 (1984) 61-69.

[7] Gouhier J., Nougier J., Nougier D., Contribution à l'étude volcanique du Cameroun ( $\ll$ ligne du Cameroun-Adamaoua »), Ann. Fac. Sci., Univ. Yaoundé, Cameroun 17 (1984) 3-48.

[8] Guiraud R., Maurin J.C., Early Cretaceaous rifts of Werstern and central Africa: an overview, Tectonophysics 213 (1992) 153-168.

[9] Hergt J.M., Peate D.W., Hawkesworth C.J., The petrogenesis of Mesozoic Gondwana low-Ti flood basalts, Earth Planet Sci. Lett. 105 (1984) 134-148.

[10] Holm P.E., The geochemical fingerprints of different ectonomagmatic environments using hygromagmatophile element abundances of tholeiitic basalts and basaltic andesites, Chem. Geol. 51 (1985) 303-323.

[11] Lee D.C, Halliday A.N., Davies G.R., Essene E.J., Fitton J.G., Temdjim R., Melt enrichment of shallow depleted mantle: a detailed petrological, trace element and isotopic study of mantle-derived xenoliths and megacrysts from the Cameroon Line, J. Petrol. 37 (2) (1996) 415-441.

[12] Le Maitre R.W., A classification of igneous rocks and glossary of terms, Blackwell Sci. Publ., Oxford, 1989, 193 p.

[13] Mc Donald G.A., Katsura T., Chemical composition of Hawaiian lavas, J. Petrol. 5 (1964) 82-133.
[14] Moreau C., Regnoult J.-M., Déruelle B., Robineau B., A new tectonic model for the Cameroon Line, Central Africa, Tectonophysics 139 (1987) 317-334.

[15] Ngounouno I., Déruelle B., Guiraud R., Vicat J.-P., Magmatismes tholéiitique et alcalin des demi-grabens crétacés de Mayo Oulo-Léré et de Babouri-Figuil (Nord du Cameroun-Sud du Tchad) en domaine d'extension continentale, C. R. Acad. Sci. Paris, série IIa 332 (soumis).

[16] Nono A., Déruelle B., Demaiffe D., Kambou R., Tchabal Nganha volcano in Adamawa (Cameroon): petrology of a continental alkaline lava series, J. Volcanol. Geotherm. Res. 60 (1994) 147-178.

[17] Regnoult J.-M., Synthèse géologique du Cameroun, Min. Mines et Énerg., Rép. Cameroun, 1986, 119 p.

[18] Sun S.S., McDonough W.F., Chemical and isotopic systematics of oceanic basalts: implications for mantle composition and processes, in : Sauders A.D., Norry M.J. (Eds.), Magmatism in the Ocean basins, Blackwell Sci. Publ., Oxford, 1989, pp. 313-345.

[19] Thornton C.P., Tuttle O.F., Chemistry of igneous rocks. I. Differentiation Index, Am. J. Sci. 258 (1960) 664-684.

[20] Toteu S.F., Geochemical characterization of the main petrographical and structural units of Northern Cameroon: implications for Pan-African evolution, J. Afr. Earth Sci. 10 (1990) 615-624. 